\title{
PROJECTIVE COMPACT DISTRIBUTIVE TOPOLOGICAL LATTICES ${ }^{1}$
}

\author{
TAE HO CHOE
}

\begin{abstract}
In the category of all compact distributive topological lattices and their continuous lattice-homomorphisms, it is shown that every projective object is either zero-dimensional or not I-compact.
\end{abstract}

By a topological lattice we mean a lattice together with a Hausdorff topology under which the two lattice operations are continuous. All terminologies and notation of lattices and category theory used in this paper are the same as in [2] and in [6], respectively.

Let $\mathscr{L}$ be a category of topological lattices and their continuous latticehomomorphisms. By a projective object $P$ in $\mathscr{L}$, we mean that, for an onto morphism $f: A \rightarrow B$ and a morphism $g: P \rightarrow B$ in $\mathscr{L}$, there exists a morphism $h: P \rightarrow A$ in $\mathscr{L}$ such that $f h=g$.

Let $I$ be the unit interval $[0,1]$ of reals with the usual topology and the usual order structure. For a topological lattice $L$, if $L$ is topologically and algebraically isomorphic with a (closed) sublattice of a product of unit intervals, then we say that $L$ is (I-compact, respectively) $I$-regular.

LemMa. Let $\mathscr{L}$ be a category of topological lattices which is closed hereditary and finitely productive. If $P$ is a connected projective object in $\mathscr{L}$ then, for every prime ideal $A$ of $P$, either $A$ or $P \backslash A$ is dense in $P$.

Proof. We may assume that $\mathscr{L}$ is nontrivial i.e., $\mathscr{L}$ has at least one nondegenerate object. Then the two element chain lattice $2=\{0,1\}$ with the discrete topology is always in $\mathscr{L}$. Clearly, the closures $A^{-}$and $(P \backslash A)^{-}$are both closed sublattices of $P$. Let $Q=\left(A^{-} \times\{0\}\right) \cup\left((P \backslash A)^{-} \times\{1\}\right)$. Then $Q$ is a closed sublattice of $P \times 2$.

Now let $j$ be the inclusion of $Q$ into $P \times 2$, and let $p$ be the projection of $P \times 2$ onto $P$. Then $p j: Q \rightarrow P$ is onto. Since $P$ is projective, for $p j$ and the identity $i$ of $P$, there exists a morphism $f: P \rightarrow Q$ in $\mathscr{L}$ such that pjf $=i$. Since $P$ is connected, either $f(P) \subset A^{-} \times\{0\}$ or $f(P) \subset(P \backslash A)^{-} \times\{1\}$. If

Received by the editors September 25, 1972.

AMS (MOS) subject classifications (1970). Primary 54F05; Secondary 18D35.

Key words and phrases. Topological lattice, category and projectivity.

${ }^{1}$ This research was supported by an NRC Grant, Canada.

(c) American Mathematical Society 1973 
$f(P) \subset A^{-} \times\{0\}$, then $P=f^{-1}\left(A^{-} \times\{0\}\right)$. On the other hand, we can show that $f^{-1}\left(A^{-} \times\{0\}\right)=A^{-}$. It suffices to show that $f^{-1}\left(A^{-} \times\{0\}\right) \subset A^{-}$. Let $x \in$ $f^{-1}\left(A^{-} \times\{0\}\right)$. Suppose that $f(x)=(y, 0) \in A^{-} \times\{0\}$. Since $p j f=i$, we have $x=y$. Thus $x \in A^{-}$. Hence $A$ is dense in $P$. Similarly, for the case that $f(P) \subset(P \backslash A)^{-} \times\{1\}, P \backslash A$ is dense in $P$.

REMARK. With a few additional conditions to those of the above lemma, it can be generalized to some other Hausdorff topological algebras of finite type as follows:

Let $\mathfrak{A}$ be a category of Hausdorff topological algebras of the same finite type which is closed hereditary and finitely productive, and let $P$ be connected projective in $\mathfrak{A}$. If

(i) the two point algebra 2 with the discrete topology is in $\mathfrak{A}$,

(ii) $A$ and $P \mid A$ are both subalgebras of $P$ and $Q=\left(A^{-} \times\{0\}\right) \cup\left((P \backslash A)^{-} \times\right.$ $\{1\})$ is a closed subalgebra of $P \times 2$ then either $A$ or $P \backslash A$ is dense in $P$.

For example, in the case of Hausdorff topological spaces (as trivial algebras) (i) and (ii) are always true and, in the case of topological semigroups, if $A$ is a prime ideal of $P$ and the two point meet semilattice with discrete topology is in $\mathfrak{A}$, then (i) and (ii) are always true.

THEOREM. Let $\mathscr{L}$ be a category of topological distributive lattices which is closed hereditary and finitely productive. Then every projective object in $\mathscr{L}$ is either totally disconnected or not I-regular.

Proof. Let $P$ be projective in $\mathscr{L}$. Suppose that $P$ is not totally disconnected. Then we have a connected component $C$ of $P$ with more than two points, and it is a closed convex sublattice of $P$ [4]. Let $J=[\alpha, \beta]$ be a nondegenerate closed interval of $C$. Then $J$ is also a closed interval in $P$, which is connected since $C$ is. Further, it is easy to see that the map $f: P \rightarrow J=[\alpha, \beta]$ defined by $f(x)=\alpha \vee(x \wedge \beta)$ is a retraction. Hence $J$ is also projective in $\mathscr{L}$. Now we show that $J$ does not have a nonconstant continuous lattice-homomorphism from $J$ into $I$. Indeed, if $g: J \rightarrow I$ is a nonconstant continuous lattice-homomorphism, then $g(J)=[r, s] \subset I$ with $r<s$. It is easy to see that $f^{-1}([r, t]) .(r<t<s)$ is a closed prime ideal of $J$, and it is neither dense in $J$ nor is its complement dense in $J$. This is a contradiction of the lemma.

COROLlaRY 1. Let $\mathscr{D}$ be the category of all compact distributive lattices. Then every projective lattice in $\mathscr{D}$ is either zero-dimensional or not I-compact.

It is known [7] that if $L$ is a compact distributive lattice then $L$ is $I$ compact iff $L$ is completely distributive. Hence by the theorem every projective lattice in the category of all compact completely distributive lattices and their continuous lattice-homomorphisms is zero-dimensional. 
It is shown [3] that, in the category of all zero-dimensional compact distributive lattices, $P$ is projective iff $P$ is a retract of the residually finite completion of a free distributive lattice.

Hence we have the following:

COROLlaRY 2. Let $\mathscr{C} \mathscr{D}$ be the category of all compact completely distributive lattices. Then every projective lattice in $\mathscr{C} \mathscr{D}$ is a retract of the residually finite completion of a free distributive lattice.

REMARK. It is known [5] that there actually exists a compact distributive lattice which is not $I$-compact. However, the author does not know whether a projective one which is not $I$-compact exists in $\mathscr{D}$. If such a projective $P$ exists in $\mathscr{D}$, then $P$ must have the following properties (i)-(iii):

(i) $P$ has a nondegenerate connected retract which has no nonconstant continuous lattice-homomorphism into $I$.

(ii) $P / \rho$, where $x \rho y$ iff $x$ and $y$ belong to the same connected component of $P$, is projective in $\mathscr{C} \mathscr{D}$.

(iii) If $P$ is connected then, for any upper (or lower) bound $x$ of a nonempty open subset of $P, x \vee P$ (or $x \wedge P$ respectively) has void interior.

\section{REFERENCES}

1. L. W. Anderson, One dimensional topological lattice, Proc. Amer. Math. Soc. 10 (1959), 715-720. MR 21 \#6401.

2. G. Birkhoff, Lattice theory, 3rd ed., Amer. Math. Soc. Colloq. Publ., vol. 25, Amer. Math. Soc., Providence, R.I., 1967. MR 37 \#2638.

3. T. H. Choe, Projective zero-dimensional compact associative distributive universal algebras, (submitted).

4. - Locally compact lattices with small lattices, Michigan Math. J. 18 (1971), 81-85. MR 43 \#8055.

5. J. D. Lawson, Lattices with no interval homomorphisms, Pacific J. Math. 32 (1970), 459-465. MR 41 \#1019.

6. B. Mitchell, Theory of categories, Pure and Appl. Math., vol. 17, Academic Press, New York, 1965. MR 34 \#2647.

7. D. P. Strauss, Topological lattices, Proc. London Math. Soc. (3) 18 (1968), 217230. MR 37 \#3532.

Department of Mathematics, McMaster University, Hamilton, Ontario, CANADA 